\title{
Avaliação do peso e da forma de carregar o material escolar de alunos que deixam seus livros na escola
}

\author{
Cleber Luiz de Sá e Silva Junior ${ }^{1}$, Cleiton Santos Sá2, Roselly Reis Batista', Mikael Ítalo Caldas ${ }^{1}$, \\ Rodrigo Gustavo da Silva Carvalho ${ }^{3}$, Lara Elena Gomes ${ }^{4}$
}

\begin{abstract}
RESUMO
O objetivo do estudo foi verificar o peso e o tipo de mochila (ou de bolsa) e como a mesma é carregada por alunos do $5^{\circ}$ ano de uma escola municipal de Petrolina-PE que deixam seus livros na escola e só carregam o restante do material necessário. A avaliação do tipo de bolsa e de como a mesma é carregada foi realizada por meio do questionário BackPEl. O peso do aluno e do material escolar foi determinado usando uma balança digital e uma balança analógica, respectivamente. Foi possível observar que quase a metade dos alunos carregava um peso acima do limite tolerável e que predominava o uso da mochila com duas alças. Entre os alunos que usavam mochila com duas alças, $82,4 \%$ a carregavam apoiada nos dois ombros. 0 estudo demonstra que, embora a escola já tenha uma estratégia para reduzir o peso do material escolar, outras medidas são necessárias.
\end{abstract}

Descritores: Postura; Criança; Dor nas costas.

\section{Evaluation of the weight and how school material is lift by students who leave their books at school}

\begin{abstract}
The purpose of the study was to verify the weight and type of bag and how it is transported by students of the 5th grade of a school in PetrolinaPE who leave their books at school and only transport the remaining material. The evaluation of the type of bag and how it is transported was conducted through questionnaire BackPEl. The weight of the student and the school material was determined using analog and digital balances, respectively. It was possible to note that almost half of the students carried a weight above the tolerable limit and it was also found a higher frequency of using of the backpack with two straps. Among students who used backpack with two shoulder straps, $82.4 \%$ carried the backpack supported on both shoulders. The study shows that, although the school already has a strategy to reduce the weight of school material, other strategies are required.
\end{abstract}

Descriptors: Posture; Child; Back Pain.

\footnotetext{
'Bacharel em Educação Física na Universidade Federal do Vale do São Francisco (UNIVASF), Petrolina, Pernambuco, Brasil.

${ }^{2}$ Acadêmico de Licenciatura em Educação Física na Universidade Federal do Vale do São Francisco (UNIVASF), Petrolina, Pernambuco, Brasil.

${ }^{3}$ Fisioterapeuta e Mestre em Educação Física pela Universidade Federal do Vale do São Francisco (UNIVASF), Petrolina, Pernambuco, Brasil.

${ }^{4}$ Mestre em Ciências do Movimento Humano pela Universidade Federal do Vale do São Francisco (UNIVASF), Petrolina, Pernambuco, Brasil.
} 


\section{Introdução}

A literatura apresenta vários fatores que podem desencadear problemas posturais na vida adulta ${ }^{1-3}$. Entre esses fatores, podem ser destacados os hábitos posturais inadequados na infância, uma vez que as estruturas musculoesqueléticas estão em desenvolvimento ${ }^{4,5}$ e os hábitos associados à escola, como o peso e a forma como 0 material escolar é carregado podem provocar alterações posturais e dor nas costas ç,6,7. $^{2}$.

Alguns estudos têm indicado que quase a metade ou mais da metade dos escolares carrega um peso superior ao limite tolerável de $10 \%$ do peso corporal ${ }^{6,8,9}$. Apesar desse resultado alarmante, os estudos que realizaram sessões educativas demonstraram melhora nesse cenário, tais como 0 aumento do número de alunos transportando a mochila de forma adequada10 e a diminuição do peso do material escolar ${ }^{10,11}$.

Desse modo, os estudos indicam que uma intervenção pedagógica proporciona o desenvolvimento de atitudes posturais mais saudáveis ${ }^{10-12}$. Por outro lado, existem também outras soluções que podem favorecer uma melhora nesse cenário: a própria escola pode tomar medidas para diminuir o peso do material escolar, por exemplo, se os livros ficarem na escola. No entanto, é necessário avaliar se essa medida é suficiente para reduzir o peso do material escolar em relação ao limite tolerável de $10 \%$ do peso corporal do aluno.

Assim, o presente estudo teve como objetivo verificar o peso do material escolar e o tipo de mochila (ou de bolsa) e como a mesma é carregada por alunos de uma escola municipal de Petrolina-PE que deixam seus livros na escola e só necessitam carregar o restante do material (lápis, borracha, caderno).

\section{Metodologia}

\section{Amostra}

A população desse estudo compreendeu alunos do $5^{\circ}$ ano de uma escola municipal de Petrolina-PE. A amostra foi composta pelos alunos que aceitaram participar do estudo e que foram autorizados pelos seus responsáveis por meio da assinatura prévia do Termo de Consentimento Livre e Esclarecido. O presente trabalho foi aprovado pelo comitê de ética sob o número de protocolo 0013/270812 CEDEP/UNIVAF (20 de julho de 2012) e recebeu autorização da Secretaria Municipal de Educação de Petrolina (16 de julho de 2012).

A partir disso, a amostra foi formada por 42 estudantes, sendo 23 meninas e 19 meninos.

A Tabela 1 ilustra as características da amostra.

Tabela 1 - Característica da amostra de acordo com idade, estatura e massa $(n=42)$.

\begin{tabular}{c|c|c|c}
\hline & Idade (anos) & Estatura $(\mathrm{m})$ & Massa $(\mathrm{kg})$ \\
\hline Média \pm desvio-padrão & $10,10 \pm 0,68$ & $1,42 \pm 0,08$ & $35,93 \pm 10,62$ \\
\hline Valor mínimo & 9,09 & 1,28 & 22,30 \\
\hline Valor máximo & 12,19 & 1,60 & 65,70 \\
\hline
\end{tabular}

\section{Aquisição dos dados}

O método utilizado para identificar o tipo de mochila (ou de bolsa) e a forma como a mesma é transportada por crianças na idade escolar foi o questionário BackPEI3, pois este apresenta validade e excelente reprodutibilidade. Para a verificação da massa dos escolares, foi utilizada uma balança digital com $0,1 \mathrm{~kg}$ de resolução (WISO, modelo W721). Já para a verificação da massa do material escolar, foi usada uma balança analógica com resolução de 0,025 $\mathrm{kg}$ (Feiticeira Ind. Utilid.Dom.Ltda). Como a balança analógica possuía uma capacidade de somente $5 \mathrm{~kg}$, a balança digital também foi usada para verificar a massa do material escolar quando este parecia exceder $5 \mathrm{~kg}$ de massa.

A coleta ocorreu na sala de aula dos alunos. Um dos pesquisadores envolvidos no estudo, inicialmente, apresentou o grupo e explicou brevemente o que iria ocorrer. Desse modo, o questionário foi aplicado e, à medida que os alunos acabavam de responder, eles guardavam todo o seu material e eram direcionados para as balanças para terem sua massa medida e, depois, para a trena, verificando a estatura ao mesmo tempo em que o material escolar era pesado. 
Essa ordem de coleta foi estabelecida, porque a escola só permitiu a liberação dos alunos para o estudo após a finalização da primeira hora de aula.

Além disso, como os pesquisadores só tiveram acesso aos alunos uma vez por semana, a massa do material escolar também foi verificada após uma semana da primeira coleta. Isso foi realizado com o intuito de verificar se a massa medida na primeira coleta seria igual a da segunda, ou seja, para ter certeza de que a massa do material escolar medida na primeira coleta fosse um valor que representasse o mais próximo possível a realidade. Também é importante destacar que, como os alunos deixavam os livros na escola e só usavam um caderno, não era esperada uma variação dos valores de massa do material escolar durante a semana.

\section{Análise dos dados}

Os dados provenientes do questionário e os valores de massa do material escolar e dos alunos foram tabulados no programa Excel (versão 2007). Para analisar a variável percentual do peso do material em relação ao peso corporal dos alunos, foi realizada uma regra de três simples, em que a massa do aluno foi considerada como 100\%, assim, o resultado foi encontrado dividindo o produto entre a massa da mochila e 100 pela massa corporal do aluno. É importante destacar que como o peso é o produto entre a massa e a gravidade, e esta é uma constante, a mesma não foi considerada no cálculo.

\section{Procedimentos estatísticos}

Foi verificada a porcentagem de respostas para cada alternativa das questões sobre o tipo de mochila (ou de bolsa) e a forma como a mesma é transportada. Já para a variável percentual do peso do material em relação ao peso corporal dos alunos, os valores de média, desvio-padrão, valores mínimo e máximo foram determinados.

Para comparar os valores de massa do material escolar entre as duas coletas, um teste t para dados pareados foi aplicado após a verificação da normalidade dos dados por meio do teste Shapiro-Wilk. Como não houve diferença entre as duas medidas, optou-se por analisar e apresentar a massa do material escolar verificada no primeiro dia.

Todos os procedimentos estatísticos foram realizados no software SPSS (versão 17.0), sendo que o nível de significância de $5 \%$ foi adotado.

\section{Resultados}

Não foi encontrada diferença entre as massas dos materiais escolares medidas em um intervalo de uma semana, $\mathrm{t}(41)=-$ $1,701, p=0,097, r=0,26$. A Tabela 2 ilustra os resultados referentes à massa do material escolar e ao peso do material escolar em relação ao peso corporal dos alunos, sendo que foi observado que, dos 42 alunos avaliados, 20 carregavam um peso superior a $10 \%$ do peso corporal e, destes, sete carregavam um peso superior a $15 \%$ do peso corporal.

A Tabela 3 ilustra os resultados para uma das questões do questionário BackPEI com intuito de avaliar o que os alunos utilizavam para carregar o material escolar. Já a Tabela 4 ilustra os resultados para outra questão do questionário BackPEI com intuito de avaliar como o aluno levava a mochila. Como essa última pergunta só foi respondida por aqueles que usavam a mochila, apenas 34 alunos responderam essa questão.

Tabela 2 - Massa do material escolar e o peso do material escolar em relação ao peso corporal dos alunos ( $n=42)$.

\begin{tabular}{c|c|c}
\hline & $\begin{array}{c}\text { Massa do material escolar } \\
(\mathbf{k g})\end{array}$ & $\begin{array}{c}\text { Peso do material escolar em } \\
\text { relação ao peso corporal (\%) }\end{array}$ \\
\hline Média \pm desvio-padrão & $3,53 \pm 0,84$ & $10,60 \pm 3,96$ \\
\hline Valor mínimo & 2,0 & 4,37 \\
\hline Valor máximo & 5,80 & 21,19 \\
\hline
\end{tabular}


Tabela 3 - Tipo de mochila (ou de bolsa) usada para carregar o material escolar $(n=42)$.

\begin{tabular}{c|c|c}
\hline & Número de alunos & Percentual \\
\hline Mochila com duas alças & 34 & $81,0 \%$ \\
\hline Mochila com uma alça & 01 & $2,4 \%$ \\
\hline Mochila com rodinhas & 04 & $9,5 \%$ \\
\hline Bolsa & 01 & $2,4 \%$ \\
\hline Pasta & 00 & $0,0 \%$ \\
\hline Outro & 02 & $4,8 \%$ \\
\hline
\end{tabular}

Tabela 4 - Forma que a mochila é carregada $(n=34)$.

\begin{tabular}{c|c|c}
\hline & Número de alunos & Percentual \\
\hline Em um ombro & 05 & $14,7 \%$ \\
\hline Nos dois ombros & 28 & $82,4 \%$ \\
\hline Outro modo/não sei & 01 & $2,9 \%$ \\
\hline
\end{tabular}

\section{Discussão}

Alguns estudos indicam que quase a metade ou mais da metade dos escolares carrega um peso superior a $10 \%$ do peso corporal $6,8,9$. Contudo, existem medidas que podem ser tomadas com o intuito de reverter esse quadro. Por exemplo, existem evidências de que intervenções educativas podem beneficiar os escolares ${ }^{10-12}$. Por outro lado, outras medidas podem ser tomadas como, por exemplo, deixar os livros na própria escola.

A partir dos resultados, pode-se observar que quase a metade dos alunos avaliados carrega o material escolar acima do limite tolerável de $10 \%$ do peso corporal. Esse resultado é semelhante com o encontrado por Stael e Lima ${ }^{6}$, os quais avaliaram 63 estudantes e notaram que 36 alunos carregavam uma carga superior a 10\% do peso corporal. Além disso, a média encontrada (Tabela 2) também é semelhante à média encontrada por outro estudo que avaliou estudantes do $1^{0}$ ao $5^{0}$ ano ${ }^{13}$. Por outro lado, Gomes e colaboradores ${ }^{8}$, após avaliarem 14 alunos de ambos os sexos com idade de 10 anos, observaram que $85,71 \%$ destes carregavam o material escolar com peso acima de $10 \%$ do peso corporal. Esse último estudo concorda com os achados de Xavier e colaboradores ${ }^{14}$, os quais avaliaram 36 crianças de ambos os sexos de 10 a 12 anos de idade e notaram que $83,3 \%$ carregavam um peso inadequado.

Além disso, quando as mochilas foram pesadas, pôde-se notar que os alunos carregavam materiais desnecessários para a aula, tais como brinquedos, garrafa de água, maquiagem, etc. Gomes e colaboradores ${ }^{8}$ também notaram que $42,85 \%$ dos estudantes transportavam objetos além do material escolar. Isso indica a possibilidade dos pais ou responsáveis não saberem o que as crianças levam para a escola. Para complementar essa hipótese, pode-se citar o trabalho de Forjuoh, Little e Schuchmann ${ }^{15}$, os quais verificaram que os pais não têm conhecimento do peso que os filhos carregam. Esses autores também observaram que alguns pais nunca verificaram o conteúdo da mochila e que os estudantes carregavam objetos além do material escolar (livros didáticos, roupas e dispositivos eletrônicos) ${ }^{15}$, o que evidencia a necessidade de um trabalho em conjunto entre os envolvidos.

Em relação ao tipo de mochila (ou de bolsa) utilizada para carregar o material escolar, o presente trabalho encontrou que predomina o uso da mochila com duas alças (Tabela 3). Esse resultado está de acordo com outros estudos8, 13,16,17. 
Por outro lado, Fernandes, Casarotto e João $0^{10}$ observaram que menos da metade dos estudantes avaliados usavam mochila, mas que esse quadro foi revertido após sessões educativas ao longo de quatro meses.

Outra variável analisada pelo presente estudo foi a forma como a mochila é transportada (Tabela 4). Foi observado que a maioria dos alunos avaliados $(82,4 \%)$ usa a mochila sustentada nos dois ombros. Esse resultado vai ao encontro de outros trabalhos ${ }^{14,17}$. É importante destacar que o hábito de carregar a mochila apoiada de forma bilateral (apoiada nos dois ombros) pode prevenir possíveis alterações posturais na infância ${ }^{1,11}$.

Mesmo encontrando resultados semelhantes com outros trabalhos, o presente estudo apresenta limitações: (1) a pequena quantidade de alunos avaliados $(n=42)$ - apesar de que alguns estudos que realizaram essa avaliação apresentam um número amostral até menor - e (2) todos os alunos eram do $5^{\circ}$ ano - em função do questionário aplicado apresentar validade para escolares a partir desse ano. Assim, como questões culturais e socioeconômicas podem afetar esses resultados ${ }^{17}$, sugere-se a continuação do próprio estudo, explorando uma região do Brasil não tão investigada como o sul e o sudeste.

Independentemente das limitações da presente investigação, os resultados demostram a importância de desenvolver estratégias para (1) identificar precocemente os fatores que contribuem para o aparecimento de desvios posturais e de dor nas costas e (2) intervir nesse processo, conscientizando e alertando alunos, pais/responsáveis e professores.

\section{Considerações Finais}

Os resultados do presente estudo indicam que a maioria dos alunos investigados utilizava mochila de duas alças apoiadas nos dois ombros. Por outro lado, os resultados indicam que é necessário alertar os escolares, responsáveis e professores sobre os riscos de transportar um peso excessivo, uma vez que deixar os livros na escola não foi capaz de garantir que todos os alunos investigados carregassem um peso inferior ao limite tolerável. Consequentemente, intervenções pedagógicas são necessárias para auxiliar na transformação desse quadro.

\section{Referências Bibliográficas}

1. NOLL M, CANDOTTI CT, ROSA BN, SCHOENELL MCW, TIGGEMANN CL, LOSS JF. Dor nas costas, hábitos posturais e comportamentais de escolares da rede municipal de ensino de Teutônia, RS. Rev Bras Cresc Des Hum. 2013;23(2):1-10. 2. RODRIGUES S, MONTEBELO MIL, TEODORI RM. Distribuição da força plantar e oscilação do centro de pressão em relação ao peso e posicionamento do material escolar. Rev Bras Fisioter. 2008;12(1):43-8.

3. NOLL M, CANDOTTI CT, VIEIRA A, LOSS JF. Back Pain and Body Posture Evaluation Instrument (BackPEI): development, content validation and reproducibility. Int J Public Health. 2013;58(4):565-72.

4. DETSCH C, LUZ AMH, CANDOTTI CT, OLIVEIRA DS, LAZARON F, GUIMARÃES LK, SCHIMANOSKI P. Prevalência de alterações posturais em escolares do ensino médio em uma cidade no Sul do Brasil. Rev Panam Salud Pública. 2008;21(4):231-8.

5. RITTER AL, SOUZA JL. Instrumento para conhecimento da percepção de alunos sobre a postura adotado no meio ambiente escolar. Rev Movimento. 2006;12(3):249-62.

6. STAEL MHS, LIMA DF. Educação postural corporal preventiva: uma abordagem a partir da medida do peso da mochila escolar em relação à massa corporal de escolares do colégio estadual de Renascença-PR. [citado em: 29 abr 2013]. Disponível em: http://www.diaadiaeducacao.pr.gov.br/portals/pde/arquivos/2372-8.pdf

7. HEUSCHER Z, GILKEY DP, PEEL JL, KENNEDY CA. The association of self-reported backpack use and backpack weight with low back pain among college students. J Manipulative Physiol Ther. 2010;33(6):432-7.

8. GOMES RM, GOUVEIA RA, MADEIRA TV, FARIA JLC. Influência da mochila escolar na postura dos alunos do ensino fundamental. Ter Man. 2011;9(44):348-352.

9. PEREIRA LM, BARROS PCC, OLIVEIRA MND, BARBOSAAR. Escoliose: triagem em escolares de 10 a 15 anos. Rev Saúde.Com. 2005;1(2):134-143.

10. FERNANDES SMS, CASAROTTO RA, JOÃO SMA. Efeitos de sessões educativas no uso das mochilas escolares em estudantes do Ensino Fundamental I. Rev Bras Fisioter. 2008;12(6):447-453.

11. BENINI J, KAROLCZAK APB. Benefícios de um programa de educação postural para alunos de uma escola municipalde Garibaldi, RS. Fisioter Pesqui. 2010;17(4):346-351. 
12. MARTÍNEZ-GONZÁLEZM, GÓMEZ-CONSECAA, MONTESINOS MDH. Programas de higiene postural desarrollados con escolares. Fisioterapia. 2008;30(5):223-30.

13. DIANAT I, JAVADIVALA Z, ALLAHVERDIPOUR H. School bag weight and the occurrence of shoulder, hand/wrist and low back symptoms among Iranian elementary schoolchildren. Health Promot Perspect. 2011;1(1):76-85.

14. XAVIER CA, BIANCHI DM, LIMA AP, SILVA IL, CARDOSO F, BERESFORD H. Uma avaliação acerca da incidência de desvios posturais em escolares. Revista Meta. 2011;3(7):81-94.

15. FORJUOH SN, LITTLE D, SCHUCHMANN JA, LANE BL. Parental knowledge of school backpack weight and contents. Arch Dis Child. 2003;82(4):261-6.

16. CANDOTTI CT, NOLL M, ROTH E. Avaliação do peso e do modo de transporte do material escolar em alunos do Ensino Fundamental. Rev Paul Pediatr. 2012;30(1):100-106.

17. DIANAT I, SORKHI N, POURHOSSEIN A, ALIPOUR A, ASGHARI-JAFARABADI M. Neck, shoulder and low back pain in secondary schoolchildren in relation to schoolbag carriage: should the recommended weight limits be gender-specific? Appl Ergon. 2014;45(2014):437-42.

\section{Cleber Luiz de Sá e Silva Junior}

Endereço para correspondência - Av. José de Sá Maniçoba, s/n, Bairro: Centro, CEP 56304-205,

Cidade: Petrolina, PE, Brasil

E-mail: cleber.junior.univasf@hotmail.com

Lattes: http://lattes.cnpq.br/9428062638619844

Cleiton Santos Sá - cleiton.sa23@yahoo.com.br

Roselly Reis Batista - rosellyreis@yahoo.com.br

Mikael Ítalo Caldas - mk_judo@hotmail.com

Rodrigo Gustavo da Silva Carvalho - rodrigo.gustavo@univasf.edu.br

Lara Elena Gomes - lara.gomes@univasf.edu.br

Enviado em 06 de março de 2014.

Aceito em 11 de dezembro de 2014. 